\title{
Change in Peak Systolic Velocity of Cavernous Artery in Response to Low Intensity Shock Waves Therapy in Diabetic Polyneuropathy Patients with Erectile Dysfunction: A Randomized Controlled Trial
}

\author{
Abdelrazak A Ahmed ${ }^{1}$ *, Wael S Shendy ${ }^{1}$, Moataz M Elsemary ${ }^{1}$, Mahmoud S Asal ${ }^{2}$, Omar M \\ Elsoghier $^{3}$
}

${ }^{1}$ Department of Physical Therapy for neuromuscular Disorders and their Surgeries, Faculty for Physical Therapy, South valley University, Egypt ${ }^{2}$ Department of Basic Science, Faculty for Physical Therapy, South valley University, Egypt ${ }^{3}$ Department of Urolog, Faculty of Medicine,South valley University Egypt

Received: 06/01/2020

Accepted: 13/04/2020

Published: 20/09/2020

\begin{abstract}
Although several reports have documented the subjective improvement of erectile function after low-intensity extracorporeal shockwave therapy (LI-ESWT) in patients with vasculogenic erectile dysfunction (ED), objective assessment data of penile hemodynamics are lacking. The aim of this research is to detect the Change in peak systolic velocity of cavernous artery in response to low intensity shock waves therapy in diabetic patients with erectile dysfunction. This study is a randomized controlled trial. Overall forty male individuals with mean age $(48.6 \pm 5.52$ years)who have erectile dysfunction after diabetes were equally divided into two separate groups. 1st Group received low intensity extracorporeal shock wave therapy (ESWT). 2nd Group received shame therapy by put head of shock wave while machine is turned off. 1st Group received low intensity extracorporeal shock wave with the following parameters: - $3000 \mathrm{SWs}$ (energy intensity of $0.09 \mathrm{~mJ} / \mathrm{mm} 2$ ) to each of five different sites of application: three areas along the penile shaft and two areas at the crural level for six weeks as a total period of treatment. Measurements of peak systolic velocity by penile duplex were performed before the treatment and after 3months. There were change in peak systolic velocity of the right and left cavernous arteries were low at baseline, indicating arterial insufficiency. After treatment, PSV significantly in the two groups; however, the posttreatment PSV was significantly higher in the SW group compared to the PFE group ( $p<0.001$, for both arteries).LIESWT is lead to significant changes in patients suffering from erectile dysfunction after diabetes as evidenced by increase in the peak systolic volume of the both right and left cavernous arteries of the shock wave group.
\end{abstract}

Keywords: Peak systolic velocity, Low intensity shock wave therapy, Erectile Dysfunction and Diabetic patients

\section{Introduction}

Erectile dysfunction (ED) is defined as the continuous inability to obtain and maintain an erection sufficient to allow satisfactory sexual intercourse. Although ED is a benign disorder, it may affect physical and psychosocial health and may have a significant impact on the quality of life $(\mathrm{QoL})$ of sufferers and their partners [1].According to the underlying causes, ED can be classified as psychogical, endocrinological, neurological, and vascular. Vascular erectile dysfunction (ED) is defined as inability to obtain or keep an erection firm enough for sexual performance due to diseases such as diabetes mellitus and atherosclerotic vascular occlusive disease [2].The onset of ED usually occurred within the first 10 years of diagnosis of type 1 and type 2diabetes in > 50\% of men affected by $\mathrm{ED}[3]$.The etiology of $\mathrm{ED}$ in diabetic patients can be vascular or neurogenic and any disruption of the primary hemodynamic events controlled by the central or peripheral neural networks that promote an erectile response will impair erectile function so both the endothelium-dependent and neural mechanisms that mediate relaxation of the smooth muscle of the corpus cavernosa impaired in diabetic patients with $\mathrm{ED}$ [4].ED is one of the most-frequent complications of diabetes mellitus (DM), which leads to a marked decrease in the quality of life and is usually treatment is difficult because of a combination of microangiopathy and peripheral diabetic neuropathy, which adversely affect the mechanism of erection[5].ED in men with diabetes has also been associated with increased age, poor glycemic control, smoking, increased alcohol intake, depression, use of specific type of medications and micro-vascular diabetic complications[6].It has been proven that $\mathrm{ED}$ and coronary artery disease $(\mathrm{CAD})$ share pathways. Endothelial dysfunction is one of the basic mechanisms has effect in the pathophysiology of vasulogenic $\mathrm{ED}$, which develops as a result of decrease in the synthesis and bioavailability of nitric oxide (NO) and subsequent atherosclerosis. Atherosclerosis leads to an impairment of the blood flow required for normal erection [7].Chronic

*Corresponding author: Abdelrazak Abdelnaeim Ahmed Mahmoud,Assistant Lecturer at the Department of Physical Therapy for Neuromuscular Disorders and their Surgeries, Faculty for Physical Therapy, South valley University.Address: Qena, Egypt. Email:dr_abdelrzak_pt@yahoo.com 
progressive impotence related directly to diabetes is the most frequent form. This form of impotence occurs years after the diagnosis of the patient. This form is progressive, irreversible and is accepted as a natural result (neuropathic, vascular or both) of the diabetes [8].Patients with diabetic and neuropathic ED have been noted to have similar frequencies of somatic and autonomic neuropathies, suggesting that neuropathy contributes significantly to diabetic ED [9].Low-intensity extracorporeal SW therapy (Li-ESWT) was used both in vitro and in vivo studies and the results shown that shock wave energy can stimulate angiogenesis. The idea of application of Li-ESWT to the penis came from animal studies in which LiESWT was applied to the myocardium of pigs, where it has been found that there was an improvement in ischemia that induced myocardial dysfunction [10].Low-intensity extracorporeal SW therapy (LIESWT) of the penis would improve penile blood flow and endothelial function by stimulating angiogenesis in the corpora [11]Consequently, it will be of importance to do a randomized controlled trial using valid and reliable measures to investigate the effect of $\mathrm{Li}$ ESWT in treatment of erectile function in diabetic polyneuropathic patients.

\section{Materials and Methods \\ 2.1 Design of the study}

The study was designed as a prospective, randomized, controlled trial. It was performed between July2018 and January 2020.

\subsection{Participants}

The study was carried out on forty outpatient male individuals with mean age ( $48.6 \pm 5.52$ years) who have erectile dysfunction after diabetes. Their age was ranging from 35 to 60 years. They were selected from South valley University hospitals. The body mass index (BMI) of those individuals not more than 32they were diagnosed with type 2 diabetes with secondary complication of erectile dysfunction. These individuals reported no other pathological conditions lead to erectile dysfunction. They showed the necessary cooperation needed to enable the investigator to secure the required data. Patients were equally divided into two separate groups. 1st Group (the study group) received the low intensity extracorporeal shock wave therapy (ESWT). 2nd Group (control group) received only shame therapy.

\subsection{Inclusive criteria}

All patients had the following characteristics; their ages ranged between 35 and 60years, they had the body mass index (BMI) of those individuals not more than 32 they were diagnosed with type 2 diabetes with secondary complication of erectile dysfunctionthe diagnosis was confirmed by using penile duplex. All patients take their medications described by their andrologyist physicians.

\subsection{Exclusive criteria}

Patients with a history of past radical prostatectomy or extensive pelvic surgery, recovering from cancer in the past 5 years, any unstable medical, psychiatric disorder, spinal cord injury and another neurological disease, penile anatomical abnormalities, clinically significant chronic hematological disease, patients with untreated hypogonadism and cardiovascular conditions that prevent sexual activity (heart attack, stroke or life-threatening arrhythmia within the previous 6 months were excluded.

\subsection{Randomization}

Every individual was informed about the nature, purpose, benefits of the research and their right to withdraw or refuse at any time. The patients were randomly assigned into 2 equal groups (control group and study group) with the use of a computer-based randomization program. No subject dropped out from the study after randomization. The patients were blinded about which group they were allocated.

\subsection{Ethical approval}

The research has complied with all the relevant national regulations and institutional policies and has followed the tenets of the Declaration of Helsinki and the Consolidated Standards of Reporting Trials and has been approved by the institutional review board at Faculty of Physical Therapy, Cairo University. No.P.T.REC/012/001983.

\subsection{Procedures}

\subsubsection{Assessment device}

The equipment used in the measurement on this research was the Penile color-coded duplex scanning machine (ultrasound with 8.4MHZ linear vascular probe (VF 15-3 trasducer, ACSON X 300 SIEMENS ultrasound) that used to get an unbiased assessment of the penile haemodynamics.

\subsubsection{Treatment devices}

The therapeutic equipment and tools used on this research were Shock wave device; ESWT device (MASTERPULS MP200, StorzMedical, Tägerwilen, Switzerland),

\subsection{Procedures}

\subsubsection{Assessment procedures}

Penile color-coded duplex scanning (Color-coded Duplex scanner Transducer: 5-10 MHz)

- Patient's position: supine

- Probe position: placed on the back of the penis

- Transversal scanning to assess the echo structure and dimensions of the cavernous bodies.

- Longitudinal scanning to identify the cavernous arteries, their patency and their hemodynamic through these parameters. (I) Peak systolic volume for right cavernous artery and (II) peak systolic volume for left cavernous artery.

Treatment Procedure: The 1st group received low intensity shock wave while the 2 nd group received shame therapy. Shock wave therapy application:The patient was placed in supine lying position receive low intensity extracorporeal shock wave with the following parameters: - 3000 SWs (energy intensity of $0.09 \mathrm{~mJ} / \mathrm{mm} 2$ ) to each of five different areas: three along the shaft of penis and two at the crural level [12].The protocol consists of 6 treatment sessions, once per week. The shock waves delivered through the applicator covering the corpora cavernosa of the penis along the penile shaft and the crura.Before the experiment, recording of the peak systolic velocity of right and left cavernous arteries for every individual were done in both groups. Treatment of ESWT application was applied one session every week for six weeks after which the second (final) penile duplex parameter (PSV) recording was taken. The pre and post experimental measurements were performed during the same time of the day to decrease the variability. The step-by-step procedure for data recording was identical to that followed during pre-experimental measurement of the penile duplex parameter (peak systolic velocity) taken before the treatment as an initial record and then after six weeks as a second last record in the two groups. 


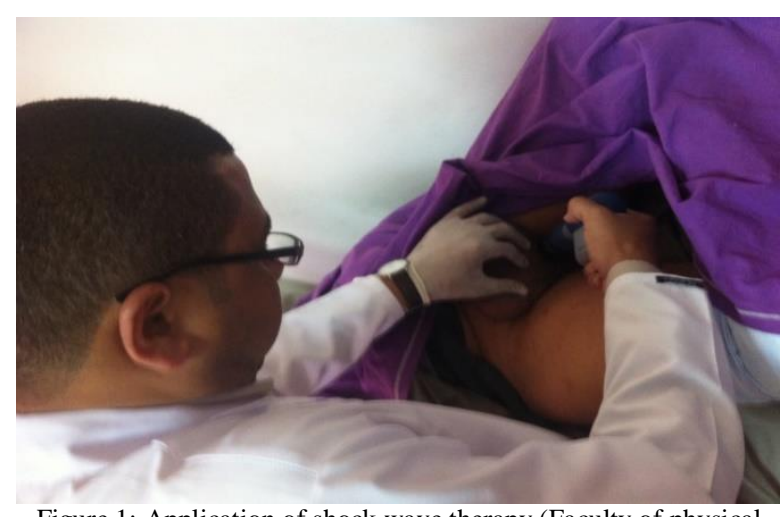

Figure 1: Application of shock wave therapy (Faculty of physical therapy south valley university2019)

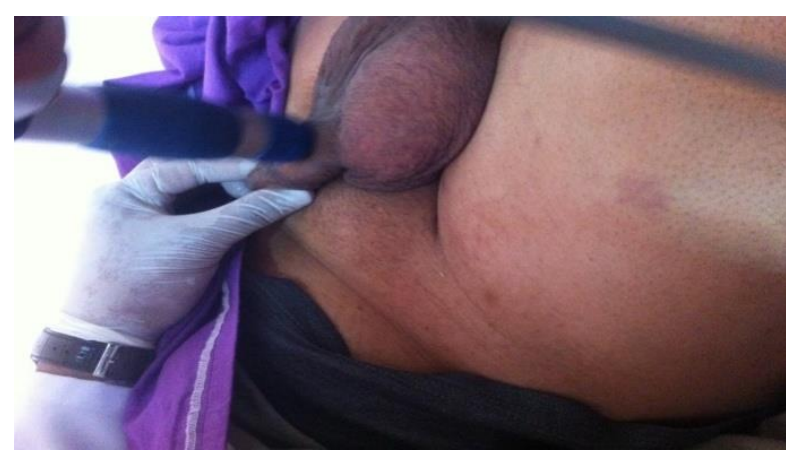

Figure 2: Application of shock wave therapy (Faculty of physical therapy south valley university 2019)

\subsubsection{Statistical analysis}

Data collected were admitted into a personal computer to analyze the statistics; the statistical analysis was conducted by using t-test to compare the patient characteristics of both groups. TheShapiro-wilk test revealed that the data were normally distributed for all dependant variables.Levrne,s test was conducted to test the homogeneity between groups. To compare the mean values of peak systolic velocity between both groups and before and after the treatment in both groups use paired $t$ test. Level of significance of significance for allstatistical testes was set at $\mathrm{P}<0.05$. with using the statistical package for social studies (SPSS) version 23 and window ( IBM SPSS, CHICAGO IL USA) for data analysis.

\subsubsection{Informed consent}

Informed consent has been obtained from all individuals included in this study

\section{Results \\ 4.1 Demographic characteristics}

Table 1 showed demographic characteristics of both groups. There was no significant difference between both groups in the mean age, weight, height and body mass index $(p<0.05)$. The mean values of peak systolic velocity for right and left cavernousarteriesshowed a statistically significant improvement $(\mathrm{p}<0.05)$ With in both groups $(\mathrm{A}$ and $\mathrm{B})$. The post-treatment comparison of both groups revealed a statistically significant increase in the mean values of peak systolic velocity for right and cavernousarteries in favor of group (A) (Table 2)

\section{Discussion}

This study demonstrated the positive effect of Li-ESWT on erectile function in patients with $\mathrm{ED}$ caused by diabetic polyneuropathy three months after treatment. Li-ESWT induced a significant increase of of penile hemodynamics, evidenced by a significant rise of PSV. Generally, the treatment was safe and well-tolerated, with no serious adverse events.In the current study, all patients were confirmed to have diabetic polyneuropathy. Doppler assessment before treatment indicated arterial insufficiency (a PSV $<25 \mathrm{~cm} / \mathrm{s}$ ) in less than half of the patients. This observation indicates that neurogenic, rather than a vasculogenic element, is operating as a cause of ED in the current series. In fact, DM-related ED is a multifactorial process that involves vascular and neurogenic causes in addition to endothelial dysfunction and nitric-oxide system disturbance [13]. However, diabetic neuropathy is believed to be a major contributing factor in the mechanism of ED in patients with type-2 DM [14]

Table 1: Participants characteristics

\begin{tabular}{lllcc}
\hline & Study group & Control group & t- value & p- value \\
\cline { 2 - 5 } & $\overline{\mathrm{x}} \pm$ SD & $\overline{\mathrm{x}} \pm$ SD & 0.56 & 0.993 \\
\hline Age (years) & $48.6 \pm 5.52$ & $47.5 \pm 5.68$ & 0.080 & 0.937 \\
Weight $(\mathbf{k g})$ & $84.52 \pm 8.98$ & $85.4 \pm 8.89$ & 0.814 & 0.355 \\
Height $(\mathbf{c m})$ & $167.53 \pm 3.58$ & $169.8 \pm 6.73$ & 0.232 & 0.827 \\
Body mass indexkg/m & $25.2 \pm 2.46$ & $26.2 \pm 2.46$ & & \\
\hline
\end{tabular}

$\overline{\mathbf{x}}$, Mean; SD, Standard deviation; p-value, Level of significance.

Table 2: Mean peak systolic velocity for right and left cavernous arteries for patient's pre and post treatment of both groups

\begin{tabular}{|c|c|c|c|c|c|c|}
\hline & \multicolumn{3}{|l|}{ Pre treatment } & \multicolumn{3}{|c|}{ Post treatment } \\
\hline & Study group & Control group & & Study group & Control group & \\
\hline & $\overline{\mathrm{X}} \pm \mathbf{S D}$ & $\overline{\mathrm{x}} \pm \mathrm{SD}$ & $P$ value & $\overline{\mathrm{x}} \pm \mathrm{SD}$ & $\overline{\mathrm{x}} \pm \mathrm{SD}$ & $P$ value \\
\hline $\begin{array}{l}\text { PSV OF } \\
\text { RTcavernous } \\
\text { artery } \mathrm{cm} / \mathrm{sec}\end{array}$ & $26.58 \pm 6.94$ & $26.2 \pm 6.06$ & 0.989 & $36.24 \pm 6.76$ & $26.7 \pm 5.70$ & $0.001 *$ \\
\hline $\begin{array}{l}\text { PSV OF } \\
\text { LTcavernous } \\
\text { artery } \mathrm{cm} / \mathrm{sec}\end{array}$ & $25.59 \pm 6.65$ & $26.60 \pm 5.75$ & 0.896 & $34.80 \pm 6.53$ & $26.38 \pm 5.68$ & $0.001 *$ \\
\hline
\end{tabular}

$\bar{X}$ : Mean, SD: Standard deviation, PSV: peak systolic velocity, level of significance:* Significant 
The neurogenic element in DM exerts its effect by inefficient nerve signaling to the corpora cavernosa. This functional alteration results in the reduction in nitric oxide (NO) load in the smooth muscles [15]. The mechanism of action of Li-ESWT is still hypothetical. Physically, shockwaves exert two effects, mechanical stress due to exposure to highpeak pressure waves and cavitations bubbles formed in liquids. These bubbles result from the vaporization of theliquid. Consequently, these cavities collapse when exposed to highpressure causing local trauma and neovascularization[16]. In vitro and in vivo studies confirmed the process of neovascularization in response to shockwave, which is believed to be a principal therapeutic mechanism in the treatment of $\mathrm{ED}[17]$. Other mechanisms are hypothesized, including NO induction [18], nerve regeneration, andstem cell proliferation[19]. In diabetic rat mode, Li-ESWT was found to affect penile neural tissue with the enhancement of neuronal NO synthase positive cells[20].Tepekoylu $\mathrm{C}$ et al. [21]found that low-intensity SW has shear stress and induces changes in membrane permeability, which leads to activation of signaling cascades, thus, improve angiogenesis,modulation of the inflammatory response,release of nitric oxide, and targeting of endothelial progenitor cells and stem cells to the active organs. Fode, $\mathrm{M}$ et al. [22] stated that two proposed mechanisms whereby LIESWT improve erectile function: Shear stress and endothelium disruption by growth and implosion of cavitations bubbles in the vessels, which result in neo angiogenesis and endothelial and neuronal nitric oxide synthesisSchuh et al. [23] investigated the effects of ex vivoshockwave treatment of nerves on subsequent Schwann cell cultures from these nerves and found consistently higher purity, proliferation rate, and expression of regenerative phenotype-associated markers (p75 neurotrophic factor receptor, glial fibrillary acidic protein, c-Jun) in pretreated Schwann cell cultures. Hence, these studies suggest an effect of shockwave therapy on nerve regeneration, which could be established by supporting Schwann cell proliferation.Assaly R, et al., [24] stated that the effect of LIST on EF was examined in an animal model. Shock wave therapyenhance nerve stimulated erection in diabetic rats, increase the smooth muscle- collagen ratio, increased the endothelial content of penile tissue and up-regulated the expression of growth factors. Qiu, X et al. [25]. Found that LIESWT are good for penile tissue regenerating by mesenchymal stem cells activation andnerve regeneration (via Schwann cells activation), and vessels, with the consequent release of pro-angiogenetic growth factors. In addition, LIESWT also enhance erectile function via nitric oxide/cGMPnondependent mechanisms.Rizk et al. [26]included randomized controlled trials, meta-analyses, and select singlearm studies on the use of Li-ESWT in the treatment of at least mild ED, with some data supporting efficacy in moderate-tosevere ED also demonstrated some benefit in specific subsets of men with vasculogenic ED (including patients with DM). Kalyvianakis et al. [27] assessed the efficacy and safety of 6and 12-treatment sessions within a 6-week treatment period and also investigated the effect of repeat treatment after a 6 - month period in a 2-phase study in patients with vasculogenic ED. The results demonstrated that re-treating patients after 6 Months could further improve EF without side effects. In addition, it was demonstrated that 12 sessions can be delivered within 6 weeks without a 3-week break period with similar clinical outcome. After discussion of the results and according to reports of the previous investigators in fields related to this study, it can be claimed that the application of LI-ESWT in erectile dysfunction in diabetic polyneuropahty patients increase peak systolic velocities of right and left cavernous arteries which result in improved erectile functions.

\section{Limitations}

The study was limited by emotional state of the patients, and the psychological condition of the patients at the time of performance which might affect the results, also, other limitation are small sample size and possible errors in measuring penile haemodynamics. So, more extensive studies assigning the efficacy of ESWT on the erectile function in diabetic polyneuropathy patients with larger sample are needed. Follow-up studies would be of great interest to detect the long-term effect of ESWT and the recurrence of erectile dysfunction.

\section{Conclusion}

Application of ESWT in erectile dysfunction in diabetic patients improve peak systolic velocity for both right and left cavernous arteries. This study demonstrated that using the ESWT is beneficial in treating diabetic patients suffering from erectile dysfunction by improving peak systolic velocity in right and left cavernous arteries.

\section{Acknowledgment}

We would like to represent our gratitude to our patients for participation in this study.

\section{Ethical issue}

Authors are aware of, and comply with, best practice in publication ethics specifically with regard to authorship (avoidance of guest authorship), dual submission, manipulation of figures, competing interests and compliance with policies on research ethics. Authors adhere to publication requirements that submitted work is original and has not been published elsewhere in any language.

\section{Competing interests}

The present study was performed in absence of any conflict of interest which was declared by the authors.

\section{Authors' contribution}

AA, WS, ME, OE and MS conceived of the study, designed the study protocol and drafted the manuscript. WE are the corresponding author and supervisor of the research. AA helped us in drafting the revised manuscript and substantively helped us to revise the manuscript. All authors have reviewed the final version of the manuscript and approve it for publication.

\section{Disclosure statement}

No author has any financial interest or received any financial benefit from this research.

\section{References}

1-Ruffo A, Capece M, Prezioso D, Romeo G, Llliano E, Romis L, Di Lauro G, Lacono F. Safety and efficacy of low intensity shockwave treatment in patients with erectile dysfunction. Int Braz J Urol. 2015; 41(5):967-74.

2-Stief C, Wespes E. Physiology and pathophysiology of Men's Sexual Arousal and Penile Erection. In F. Montorsi, R. Basson, G Adaikan, E. Becher, A. Clayton, F. Giuliano, S. Khoury, I Sharlip,(Edit). Sexual Medicine Sexual Dysfunctions in Men and Women. 3rd International Consultation on Sexual Medicine Paris; Health Publication Ltd. 2010. 
3-Vinik A, Richardson D. Erectile dysfunction in diabetes. Diabetes Reviews 1998; 6:16-33.

4-Eardley I. Pathophysiology of erectile dysfunction. $\mathrm{Br} J$ Diabetes Vasc Dis 2002; 2:272-6.

5-Malavige LS, Levy JC. Erectile dysfunction in diabetes mellitus. J Sex Med 2009; 6:1232-1247.

6-Klingler HC, Kratzik C, Pycha A, Marberger M. Value of power Doppler sonography in the investigation of erectile dysfunction. Eur Urol. 1999 Oct; 36 (4):320-6. 7.

7-Kefi A, Demir Ö, Seçil M, Esen A.A. Doppler ultrasonography in erectile dysfunction patients with risk factors. Turk J Urol. 2005; 31:202-6.

8-Saenz de Tejada I, Goldstein I. Diabetic penile neuropathy. Urol Clin North Am. 1988 Feb;15(1):17-22.

9-Hecht MJ, Neundorfer B, Kiesewetter F, Hilz MJ. Neuropathy is a major contributing factor to diabetic erectile dysfunction. Neurol Res 2001; 23: 651-4.

10-Nishida T, Shimokawa H, Oi K, Tatewaki H, Uwatoku T, Matsumoto Y, Kajihara N, Eto M, Matsuda T, Sunagawa K. Extracorporeal cardiac shock wave therapy markedly ameliorates ischemia-induced myocardial dysfunction in pigs in vivo. Circulation 2004; 110:3055-61.

11-Gruenwald I, Kitrey N.D, Appel B, and Vardi Y. Low-Intensity Extracorporeal Shock Wave Therapy in Vascular Disease and Erectile Dysfunction: Theory and Outcomes. Sex Med Rev. 2013; 1:83-90.

12-Vardi Y, Appel B, Jacob G, Massarwi O, Gruenwald I. Can lowintensity extracorporeal shockwave therapy improve erectile function? A 6-month follow-up pilot study in patients with organic erectile dysfunction. Eur Urol. 2010; 58:243-248.

13- Phé V, Rouprêt M. Erectile dysfunction and diabetes: a review of the current evidence-based medicine and a synthesis of the main available therapies. Diabetes Metab. 2012;38:1-13.

14- Hecht MJ, Neundörfer B, Kiesewetter F, Hilz MJ. Neuropathy is a major contributing factor to diabetic erectile dysfunction. Neurol Res. 2001;23:651-4.

15- Yafi FA, Jenkins L, Albersen M, Corona G, Isidori AM, Goldfarb S, et al. Erectile dysfunction. Nat Rev Dis Primer. 2016;2:16003.

16- Young Academic Urologists Men's Health Group, Fode M, Hatzichristodoulou G, Serefoglu EC, Verze P, Albersen M. Lowintensity shockwave therapy for erectile dysfunction: is the evidence strong enough? Nat Rev Urol. 2017;14:593-606.

17- Bongrazio M, Da Silva-Azevedo L, Bergmann EC, Baum O, Hinz $\mathrm{B}$, Pries AR, et al. Shear stress modulates the expression of thrombospondin-1 and CD36 in endothelial cells in vitro and during shear stress-induced angiogenesis in vivo. Int $\mathbf{J}$ Immunopathol Pharmacol. 2006;19:35-48.

18- Gotte G, Amelio E, Russo S, Marlinghaus E, Musci G, Suzuki H. Short-time non-enzymatic nitric oxide synthesis from L-arginine and hydrogen peroxide induced by shock waves treatment. FEBS Lett. 2002;520:153-5

19- Sokolakis I, Dimitriadis F, Teo P, Hatzichristodoulou G, Hatzichristou D, Giuliano F. The Basic Science Behind LowIntensity Extracorporeal Shockwave Therapy for Erectile Dysfunction: A Systematic Scoping Review of Pre-Clinical Studies. J Sex Med. 2019;16:168-94.

20- Qiu X, Lin G, Xin Z, Ferretti L, Zhang H, Lue TF, et al. Effects of low-energy shockwave therapy on the erectile function and tissue of a diabetic rat model. J Sex Med. 2013;10:738-46.

21- Tepekoylu C, Polzl L, Lobenwein D, Graber M, Blumer M, Kirchmair E, Kirchmair R, Davidson S, Paulus P, Grimm M, Holfeld J. Shock waves induce angiogenesis via exosome release. Eur Heart J Acute Cardiovasc Care 5: 85-86, 2016.

22-Fode, M.; Hatzichristodoulou, G.; Serefoglu, E.C.; Verze, P.; Albersen, M. Low-intensity shockwave therapy for erectile dysfunction: Is the evidence strong enough? Nat. Rev. Urol. 2017, 14, 593-606.

23-Schuh, C., Hausner T. \& Redl H. R. A therapeutic shock propels Schwann cells to proliferate in peripheral nerve injury. Brain Circul. 2016 2, 138

24-Assaly R, Laurin M, Gorny D et al: Low intensity extracorporal shock waves therapy improves erectile function in diabetic type II rats independently of NO/cGMP pathway. Presented at annual meeting of American Urological Association, New Orleans, Louisiana, May 15-19, 2015, abstract 15-6311.
25-Qiu, X.; Lin, G.; Xin, Z; Ferretti, L. Zhang, H.; Lue, T.F.; Lin, C.S. Effects of low-energy shockwave therapy on the erectile function and tissue of a diabetic rat model. J. Sex. Med. 2013, 10, 738-746.

26-Rizk PJ, Krieger JR, Kohn TP, et al. Low-intensity shockwave therapy for erectile dysfunction. Sex Med Rev 2018; 6: 624-630.

27-Kalyvianakis D, et al. Low-intensity shockwave therapy for erectile dysfunction: A randomized clinical trial comparing 2 treatment protocols and the impact of repeating treatment. J Sex Med 2018 15:334-345. 